\title{
On Sum Rate Maximization Study for Cellular-Connected UAV Swarm Communications
}

\author{
Bin Yang ${ }^{* \dagger}$, Tarik Taleb ${ }^{\dagger \dagger}$, Guilin Chen* \\ *School of Computer and Information Engineering, Chuzhou University, China \\ ${ }^{\dagger}$ School of Electrical Engineering, Aalto University, Finland \\ ${ }_{\ddagger}$ Centre for Wireless Communications, University of Oulu, Finland \\ Email: yangbinchi@gmail.com, tarik.taleb@aalto.fi, glchen@chzu.edu.cn
}

\begin{abstract}
The integration of cellular networks and unmanned aerial vehicle (UAV) swarm communications is expected to be a promising technology to provide ubiquitous network connectivity for various UAV assisted Internet of Things (IoT) applications. To support these IoT applications with stringent requirement of rate performance, this paper explores the maximum sum rate performance for the cellular-connected UAV swarm communications. The sum rate maximization can be formulated as a nonlinear and nonconvex optimization problem with the constraints of transmit power of UAVs, elevation angle, azimuth angle and height of antenna array equipped at base station (BS). According to the Karush-Kuhn-Tucker (KKT) optimality conditions and the standard interference function, we propose an iterative algorithm to solve the problem, wherein the problem is transformed into a concave optimization problem by utilizing the rate approximation and logarithmic transformations. The iterative algorithm is proved to converge to a global solution for the approximated concave optimization problem. Finally, simulation results are provided to indicate the effect of some important system parameters on the sum rate performance in the system.
\end{abstract}

Index Terms-IoT, UAV swarm, cellular networks, sum rate, power control, directional antennas.

\section{INTRODUCTION}

Owing to distinctive advantages of unmanned aerial vehicles (UAVs) such as high flexibility, cost-effective and swift deployment, UAV communications have become appealing solutions for widespread Internet of Things (IoT) applications including video streaming transmission, remote sensing, search and rescue communications, etc [1], [2]. Particularly, UAV swarm communications can cooperate with each other to perform complex tasks by overcoming the disadvantages of limited power and the number of on-board sensors for a single UAV. However, available UAV communications mainly use unlicensed spectrum (e.g., 2.4 GHZ band) to send simple peer-to-peer messages. Such communications have the features of low rate, unreliability and short coverage range, which are a serious obstacle to widely deploy UAV assisted communication systems [3], [4].

To overcome the obstacle for realizing their large-scale deployment, an appealing solution is to integrate UAVs into cellular networks guaranteeing the communication performance with high rate, reliability and remote data transmission. For the cellular-connected UAV communications, UAVs can use the licensed spectrum of cellular networks and ubiquitous ground base stations (BSs) to achieve long-distance communication services in comparison with the traditional UAV communications operated over the unlicensed spectrum.

A comprehensive understanding on performances of UAV communications is critical to support their various applications [5]-[7], [7], [8]. Available works mainly examine the performances in terms of rate, sum rate, max-min rate, coverage and energy efficiency for the UAV communications without the ground BSs, whereby UAVs act as either aerial BSs or user equipments (UEs). The works aim to optimize the following parameters to improve the performances for UAV communications: UAV altitude [9], UAV trajectory or UAV placement [10], [11], joint UAV trajectory and power allocation [12], joint UAV trajectory and communication scheduling [13], joint UAV altitude and antenna beamwidth [14], joint UAV trajectory, power allocation and communication scheduling [15], joint UAV trajectory, and power allocation and bandwidth [16].

Only some initial works are devoted to the study of performances for the cellular-connected UAV communications in terms of rate, sum rate, coverage, mission completion time by setting different parameters such as UAV cache size and density [17], UAV trajectory [18], joint power allocation and beamforming [19], joint UAV speed and channel allocation [20], and joint power allocation and UAV placement [21]. In these works, power allocation and bemforming are two importance methods to enhance the communication performances. An increase of transmit power can increase the received signal strength at a receiver while incurring severe interference. On the other hand, beamforming technique is promising to mitigate the interference. Recently, we investigate the performances for millimeter wave (mmWave) cellularconnected UAV swarm communications with angle-dependent directional antennas in terms of sum rate, fairness index, maxmin rate and proportional fairness, whereby the UAVs and BS use fixed transmit power to transmit messages at mmWave bands [22]. However, the performances are still unknown for the traditional cellular-connected UAV swarm communications operated at sub- 6 GHZ bands with the constraints of variable UAV transmit power and beamforming of antenna arrays.

Motivated by this observation, by jointly optimizing the 
power allocation and beamforming, this paper investigates the fundamental performance of maximum sum rate for the cellular-connected UAV swarm communications with sub-6 GHZ bands, where each UAV and BS are equipped more realistic angle-dependent directional antenna arrays. To this end, we formulate the sum rate maximization as an optimization problem and solve it. This is the first work to jointly optimize the power allocation and elevation angle, azimuth angle and height of antenna array associated with beamforming to maximize the sum rate for the cellular-communications UAV swarm communications. The main contributions are summarized as follows.

- We formulate the sum rate maximization as a nonconvex optimization problem subject to the constraints of UAV transmit power, elevation angle, azimuth angle and height of antenna array at BS. Sepcially, a more realistic angledependent directional antenna array is used to model the three dimensional (3D) antenna beamforming gain for cellular-enabled UAV swarm communications.

- To solve the nonconvex optimization problem, it is transformed into a concave optimization problem by rate approximation and logarithmic transformations. Then, we propose an iterative algorithm to solve the concave optimization problem according to the Karush-Kuhn-Tucker (KKT) optimality conditions and the standard interference function results, which is proved to converge to a global optimal solution.

- Finally, simulation results are provide to indicate the effect of some key system parameters on the sum rate, and also to show our new findings.

The remainder of the paper is organized as follows. The system model is introduced in Section II. The problem formulation and solution of sum rate maximization are presented in Sections III. Section IV presents the simulate results. Finally, Section V concludes the paper.

\section{SySTEM Models}

\section{A. Communication Model}

As illustrated in Fig. 1, we consider an uplink transmission scenario where a UAV swarm communicates with a BS. These UAVs are spatially distributed according to a homogeneous Poisson point process $\Phi$ of intensity $\lambda_{U}$, and hover at the same altitude $H_{U}$. We use $|\Phi|$ to denote the number of UAVs, and use $P_{1}, \ldots, P_{|\Phi|}$ to denote the transmit powers of the $|\Phi|$ UAVs. Particularly, $P_{i}$ is the transmit power of the $i$ th UAV with a constraint of maximum value $P_{\max }$. These UAVs can adjust the power vector $\mathbf{P}=\left[P_{1}, \ldots, P_{|\Phi|}\right]^{\mathrm{T}}$ to optimize the system performance.

\section{B. Directional Beamforming}

As shown in Fig. 1, both UAVs and BS use 3D directional antenna arrays to perform directional beamforming for message transmission. To maximize the antenna array gain at UAVs denoted by $G_{M}^{U}$, each UAV can adjust its boresight direction of the antenna array towards the BS receiver. On the other hand, we consider the mainlobe antenna gain and

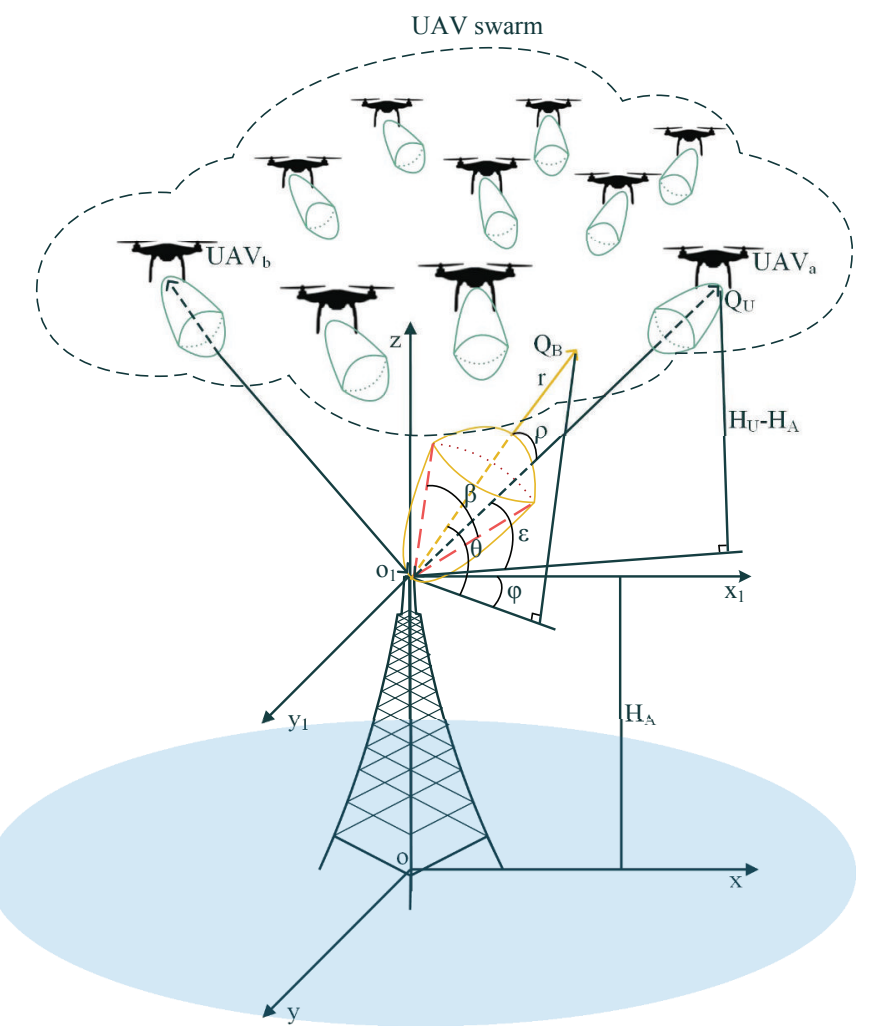

Fig. 1. Communication model consisting of a BS and a UAV swarm, where the UAVs perform beamforming with the BS

sidelobe gain at the BS. Specially, the sidelobe gain is generally much lower than the mainlobe gain due to small radiation power in the sidelobes. Thus, we assume that the sidelobe gain is a small constant $G_{S}^{B}$. Based on [23], the mainlobe gain $G_{M}^{B}(\rho)$ can be written as

$$
G_{M}^{B}(\rho)=D_{0}(\tau) \cos (\rho)
$$

where $\tau$ denotes a directivity parameter of the antenna array, $D_{0}(\tau)$ is the maximum antenna directivity, $\rho$ denotes the radiation angle between the antenna boresight direction $\overrightarrow{o_{1} Q_{B}}$ at the BS and the radiation vector $\overrightarrow{o_{1} Q_{U}}$ from the antenna location at the $\mathrm{BS}$ to $\mathrm{UAV}_{a}$ as shown in Fig. 1 , and $\cos (\rho)$ is the antenna radiation efficiency. We can see from formula (1) that $G_{M}^{B}(\rho)$ depends on $\tau$ and $\rho$, and is also symmetric along the boresight direction.

We need to calculate the unknown $D_{0}(\tau)$ and $\rho$ defined in formula (1). We first determine the maximum antenna directivity $D_{0}(\tau)$ as

$$
D_{0}(\tau)=\frac{4 \pi}{\Omega_{A}(\tau)}
$$

According to [24], the beam solid angle $\Omega_{A}(\tau)$ of the antenna 
array is expressed as

$$
\begin{aligned}
\Omega_{A}(\tau) & =\int_{0}^{2 \pi} \int_{0}^{\pi} U_{\tau}(\rho) \sin (\rho) d \rho d \varphi \\
& =\int_{0}^{2 \pi} \int_{0}^{\frac{\pi}{2}} \frac{\cos (\rho)}{\tau} \sin (\rho) d \rho d \varphi \\
& =\frac{\pi}{2 \tau}
\end{aligned}
$$

where we use $U_{\tau}(\rho)=\cos (\rho) / \tau$ to denote the normalized radiation intensity of the antenna array at the $\mathrm{BS}$, and neglect the backlobe, i.e., $U_{\tau}(\rho)=0$ for $\pi / 2 \leq|\rho| \leq \pi$, due to the fact that the backlobe antenna gain is quite small. As shown in Fig. 1, we can also obtain the half-power beamwidth $\beta$ (beamwidth for short) as a function of $\tau$. Since the mainlobe antenna gain is half of the maximum directivity gain at the angle $\beta / 2$, i.e., $U_{\tau}(\beta / 2)=1 / 2$, we get

$$
\beta=2 \arccos (\tau / 2),
$$

where $0<\tau<2$. We can see from formulas (2) and (3), a larger $\tau$ leads to a larger antenna directivity and a smaller beamwidth, which implies that the signal from mainlobe covers a smaller area on the air.

Finally, we calculate the radiation angle $\rho$. As shown in Fig. 1, using the operation of dot product, $\rho$ is determined as

$$
\rho=\arccos \frac{\overrightarrow{o_{1} Q_{B}} \cdot \overrightarrow{o_{1} Q_{U}}}{\left|\overrightarrow{o_{1} Q_{B}}\right|\left|\overrightarrow{o_{1} Q_{U}}\right|},
$$

where the dot - denotes the operation of dot product, and $|\vec{X}|$ denotes the square root of vector $\vec{X}$. We use $o_{1}=$ $\left(0,0, H_{A}\right)$ to denote the antenna location at the $\mathrm{BS}$, use $Q_{B}=\left(r \cos \theta \cos \varphi, r \cos \theta \sin \varphi, r \sin \theta+H_{A}\right)$ to denote the intersection point between vector $\overrightarrow{o_{1} Q_{B}}$ and sphere with center $o_{1}$ and radius $r$, and use $Q_{U}=\left(x_{U}, y_{U}, H_{U}\right)$ to denote the location of $\mathrm{UAV}_{a}$. Thus, we can obtain $\overrightarrow{o_{1} Q_{B}}=$ $(r \cos \theta \cos \varphi, r \cos \theta \sin \varphi, r \sin \theta)$ and $\overrightarrow{o_{1} Q_{U}}=\left(x_{U}, y_{U}, H_{U}-\right.$ $\left.H_{A}\right)$.

\section{Channel Model}

Due to the high altitude of the UAVs, we consider that the channels from each UAV to the BS are mainly dominated by line-of-sight (LoS) links. Note that in practice, the channels for UAV communications are more likely to be the LoS links compared with the channels for terrestrial user communications. We assume the general power law path loss model and Nakagami- $m$ fading for the LoS links. Specifically, for a UAV transmitter $i$ with transmit power $P_{i}$, the received signal power $S_{i}$ at the BS is given by

$$
S_{i}=P_{i} G^{B}\left(\rho_{i}\right) G_{M}^{U} h_{i}\left|L_{i}\right|^{-\alpha},
$$

where $h_{i}$ denotes the fading coefficient of the $i$ th LoS link, following a Nakagam- $m$ distribution with parameter $m,\left|L_{i}\right|$ denotes the distance of the $i$ th link, $\alpha>2$ denotes the path loss exponent, and $G^{B}\left(\rho_{i}\right)$ denotes the corresponding antenna gain under the radiation angle $\rho_{i}$. The $G^{B}\left(\rho_{i}\right)$ is determined as

$$
G^{B}\left(\rho_{i}\right)= \begin{cases}G_{M}^{B}\left(\rho_{i}\right) & \text { if } 0 \leq \rho_{i} \leq \beta, \\ G_{S}^{B} & \text { if } \rho_{i}>\beta .\end{cases}
$$

We consider a multi-channel environment for the communication scenario. The frequency resources of the scenario are evenly divided into $N$ orthogonal channels, and the bandwidth of each channel is $W$ MHZ. Each UAV randomly selects a channel from these orthogonal channels for message transmission. The interference is caused by the UAVs sharing the same channel while there is no interference among the UAVs using different channels. In addition, the channel noise is additive white Gaussian noise with variance $\sigma^{2}$.

\section{SINR of the BS}

Based on the above antenna gain and channel model, the signal-to-interference-plus-noise ratio (SINR) received at the $\mathrm{BS}$ receiver from a UAV $i$ can be expressed as

$$
\operatorname{SINR}_{i}\left(\mathbf{P}, \theta, \varphi, H_{A}\right)=\frac{S_{i}}{I_{i}+\sigma^{2}},
$$

where $I_{i}$ denotes the interference seen by the UAV $i$ at the BS. The $I_{i}$ is determined as

$$
I_{i}=\sum_{j \in \Phi_{i}^{*}, j \neq i} P_{j} G^{B}\left(\rho_{j}\right) G_{M}^{U} h_{j}\left|L_{j}\right|^{-\alpha},
$$

where $\Phi_{i}^{*}$ denotes the set of the UAV transmitters using the same channel with the UAV $i$.

The rate $\gamma_{i}$ of the LoS link can also be determined as

$$
\gamma_{i}=W \log _{2}\left(1+\operatorname{SINR}_{i}\left(\mathbf{P}, \theta, \varphi, H_{A}\right)\right) .
$$

\section{Problem Formulation and Solution OF Sum RATE MAXIMIZATION}

In this section, we first formulate sum rate maximization as an optimization problem, and then propose an algorithm to solve it.

\section{A. Problem Formulation}

Our objective is to maximize the sum rate by jointly optimizing the transmit power $P_{i}$ and antenna parameters like the elevation angle $\theta$, azimuth angle $\varphi$ and height $H_{A}$. We formulate the sum rate maximization as the following optimization problem.

$$
\begin{array}{ll}
\max _{\theta, \varphi, H_{A}, \mathbf{P}} & \sum_{i \in \Phi} \gamma_{i}, \\
\text { s.t. } & 0 \leq P_{i} \leq P_{\max }, \\
& 0 \leq \theta \leq \pi, \\
& 0 \leq \varphi \leq 2 \pi, \\
& 0 \leq H_{A} \leq H_{\max },
\end{array}
$$

where $P_{\max }$ and $H_{\max }$ denote the maximum transmit power of each UAV and the maximum height of antenna array, respectively. Constraint (11b) represents that the range of transmit power, and constraints (11c),(11d) and (11e) give the range of these antenna parameters. 


\section{B. Solution of Sum Rate Maximization}

The sum rate maximization is a nonlinear and nonconvex optimization problem, and thus it is NP-hard and generally hard to solve. To solve it, we first transform it into a concave optimization problem by replacing $\log _{2}(1+$ $\left.\operatorname{SINR}_{i}\left(\mathbf{P}, \theta, \varphi, H_{A}\right)\right)$ with $\log _{2}\left(\operatorname{SINR}_{i}\left(\mathbf{P}, \theta, \varphi, H_{A}\right)\right)$ and using logarithmic transformations of $\mathbf{P}, \theta, \varphi$ and $H_{A}$. According to the Karush-Kuhn-Tucker (KKT) optimality conditions and the standard interference function results introduced in [25], an iterative algorithm is then proposed to solve the concave optimization problem, which converges to a globally optimal solution.

We use $\mathbf{P}^{*}, \theta^{*}, \varphi^{*}, H_{A}^{*}$ to denote the base 2 logarithmic transformations of $\mathbf{P}, \theta, \varphi$ and $H_{A}$, and use $P_{i}^{*}$ to denote the $i$ th element of $\mathbf{P}^{*}$. Then, the optimization problem in (11) can be approximated as

$$
\begin{array}{ll}
\max _{\theta^{*}, \varphi^{*}, H_{A}^{*}, \mathbf{P}^{*}} & \sum_{i \in \Phi} W \log _{2}\left(\operatorname{SINR}_{i}\left(\mathbf{P}^{*}, \theta^{*}, \varphi^{*}, H_{A}^{*}\right)\right), \\
\text { s.t. } & P_{i}^{*} \leq \log _{2}\left(P_{\max }\right), \\
& \theta^{*} \leq \log _{2}(\pi), \\
& \varphi^{*} \leq \log _{2}(2 \pi), \\
& H_{A}^{*} \leq \log _{2}\left(H_{\text {max }}\right),
\end{array}
$$

when the $\operatorname{SINR}_{i}\left(\mathbf{P}^{*}, \theta^{*}, \varphi^{*}, H_{A}^{*}\right)$ is much larger than one. This approximation is reasonable because the value of sum rate is mainly determined by the link rates with high SINR. For each fixed setting of $\theta^{*}, \varphi^{*}$ and $H_{A}^{*}$, the sum rate maximization with power constraint in (12a) and (12b) is a concave optimization problem.

Since $P_{i}=2^{P_{i}^{*}}$, we further obtain a fixed point iteration that

$$
\begin{aligned}
& P_{i}(\omega+1)= \\
& \min \left\{\frac{1}{\sum_{k \in \Phi_{i}^{*}, k \neq i} \frac{G^{B}\left(\rho_{i}\right) G_{M}^{U} h_{i}\left|L_{i}\right|^{-\alpha}}{\sum_{j \in \Phi_{i}^{*}, j \neq k} P_{j}(\omega) G^{B}\left(\rho_{j}\right) G_{M}^{U} h_{j}\left|L_{j}\right|^{-\alpha}+\sigma^{2}}},\right. \\
& \left.P_{\text {max }}\right\},
\end{aligned}
$$

where $P_{i}(\omega)$ denotes the value of $P_{i}$ in the $\omega$ th iteration.

Based on the fixed point iteration, we propose an algorithm to solve the optimization problem summarized in following Algorithm 1.

\footnotetext{
Algorithm 1 Solution of sum rate maximization:

1. Input: Locations of UAVs and BS, flying attitude of UAV $H_{U}$, channel bandwidth $W$, antenna sidelobe gain $G_{S}^{B}$ at BS, antenna gain $G_{M}^{U}$ at each UAV, noise power $\sigma^{2}$, and maximum sum rate $T_{\max }$.

2. Output: $T_{\max }$.

3. Initialize $T_{\max }=0, \omega=0, P_{i}(\omega)=P_{\max }$, and step size $\lambda_{\theta}, \lambda_{\varphi}$ and $\lambda_{H}$.
}

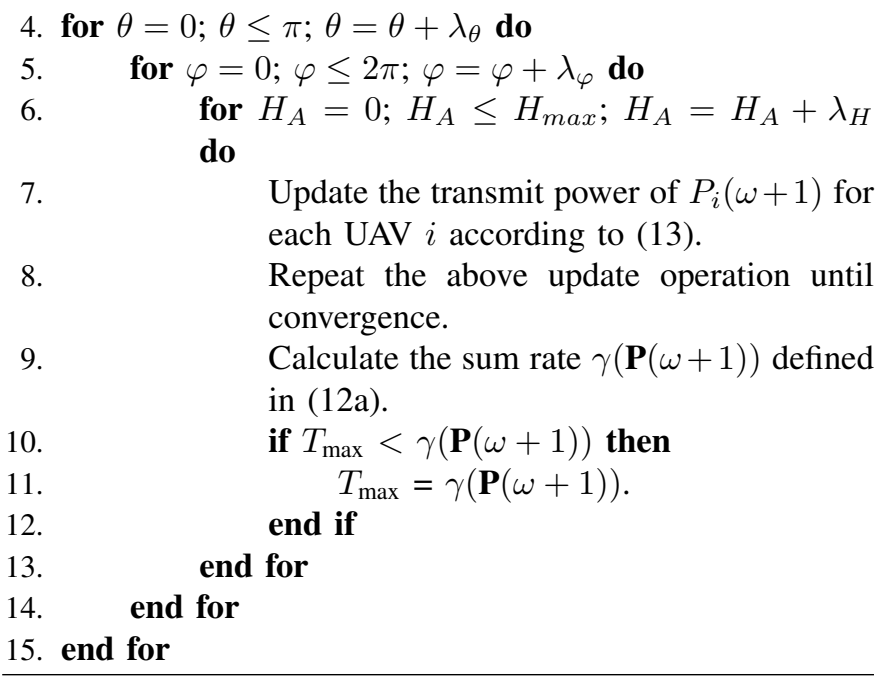

It is notable that starting from any initial transmit power $\mathbf{P}(0)$, Algorithm 1 converges to an optimal solution of the optimization problem.

TABLE I

SYSTEM PARAMETERS

\begin{tabular}{|l|l|}
\hline Parameters & Values \\
\hline \hline scenario area & $3.6 \times 10^{5} \mathrm{~m}^{2}$ \\
\hline Density of UAV $\lambda_{U}$ & $10^{-4} \mathrm{UAVs} / \mathrm{m}^{2}$ \\
\hline Altitude of UAV $H_{U}$ & $300 \mathrm{~m}$ \\
\hline Maximum transmit power of UAV $P_{\max }$ & $500 \mathrm{~mW}$ \\
\hline Number of channels $N$ & 10 \\
\hline Bandwidth of each channel $W$ & $100 \mathrm{MHZ}$ \\
\hline Maximum height of antenna array $H_{\max }$ & $120 \mathrm{~m}$ \\
\hline Directivity parameter of antenna array $\tau$ & 1 \\
\hline Antenna array gain of sidelobe $G_{S}^{B}$ & $-20 \mathrm{~dB}$ \\
\hline Path loss exponent of channel $\alpha$ & 2 \\
\hline Noise power $\sigma^{2}$ & $-90 \mathrm{dBm}$ \\
\hline
\end{tabular}

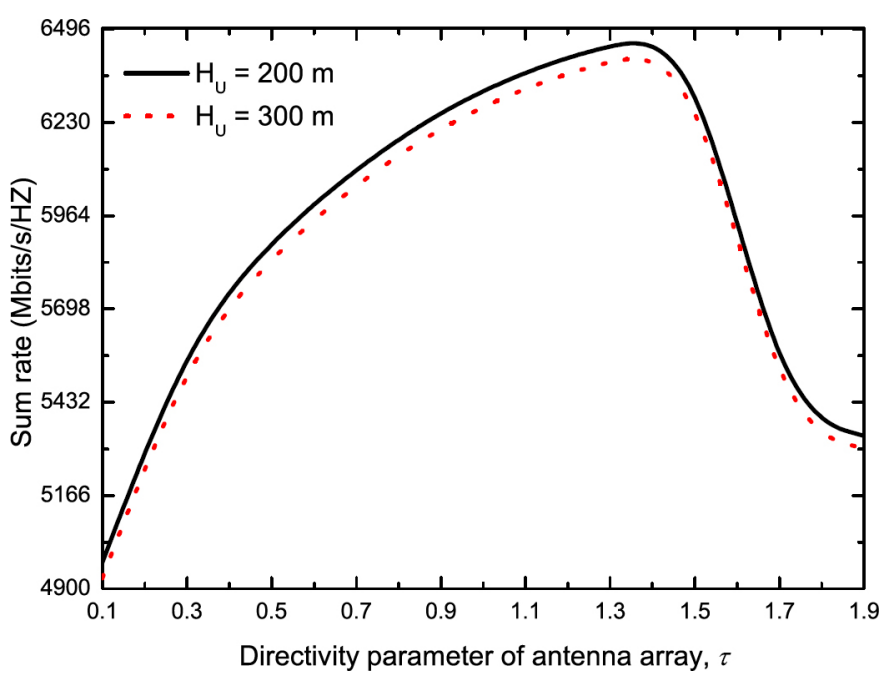

Fig. 2. The effect of directivity parameter of antenna array $\tau$ on sum rate

\section{Simulation Results}

This section provides simulation results to explore the effect of some important system parameters on the maximum sum 


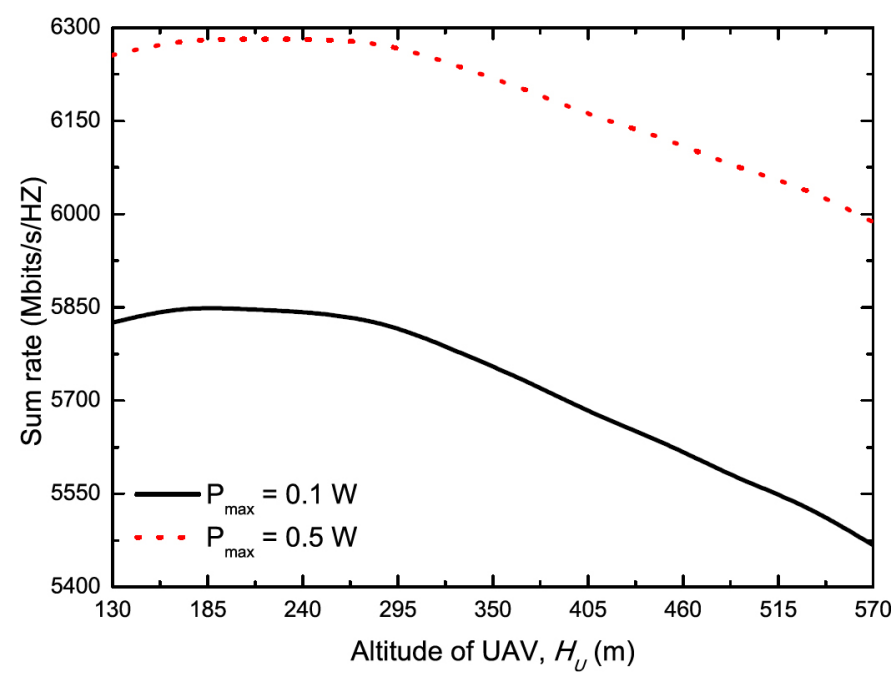

Fig. 3. The effect of altitude of UAV $H_{U}$ on sum rate

rate, which is obtained by Algorithm 1. The system parameters include the altitude of UAV $H_{U}$, maximum transmit power of UAV $P_{\text {max }}$, directivity parameter $\tau$ and maximum height $H_{\max }$ of antenna array, which are set in Table I, unless otherwise specified.

\section{A. Effect of $\tau$ On Sum Rate}

We first investigate the effect of the directivity parameter of antenna array $\tau$ on the sum rate under the sum rate maximization optimization problem for the scenario of altitude of UAV $H_{U}=\{200,300\}$ m. Fig. 2 illustrates how the sum rate varies with $\tau$. We can see from Fig. 2 that for each fixed setting of $H_{U}$, the sum rate first increases and then decreases as $\tau$ increases. This is mainly due to the following reasons. According to the formula (4), we know that an increase of $\tau$ results in the decrease of the antenna beamwidth $\beta$. This means that a small $\tau$ corresponds to a big $\beta$, so for a small $\tau$, the antenna mainlobe of BS can cover the area where most of UAVs hover. Besides, based on the formulas (1), (2) and (3), the antenna mainlobe gain $G_{M}^{B}(\rho)$ at the BS increases as $\tau$ increases, which leads to the increase of the rate of most links. Thus, for a small $\tau$, an increase of $\tau$ leads to the increase of sum rate. As $\tau$ continues to increase up to more than a threshold, more UAVs hover in the area covered by the antenna sidelobe with a very small gain, which leads to the decrease of sum rate.

\section{B. Effect of $H_{U}$ On Sum Rate}

We proceed to investigate the effect of altitude of UAV $H_{U}$ on the sum rate for the scenario of maximum UAV transmit power $P_{\max }=\{0.1,0.5\}$ W. Fig. 3 summarizes how the sum rate varies with $H_{U}$. It can be observed from Fig. 3 that for a fixed setting of $P_{\max }$, as $H_{U}$ increases, the sum rate first increases, then keeps unchanged, and finally decreases. This can be explained as follows. The increase of $H_{U}$ has two-fold effect on the sum rate. A big $H_{U}$ results in a large coverage of antenna mainlobe at BS with a high antenna gain.

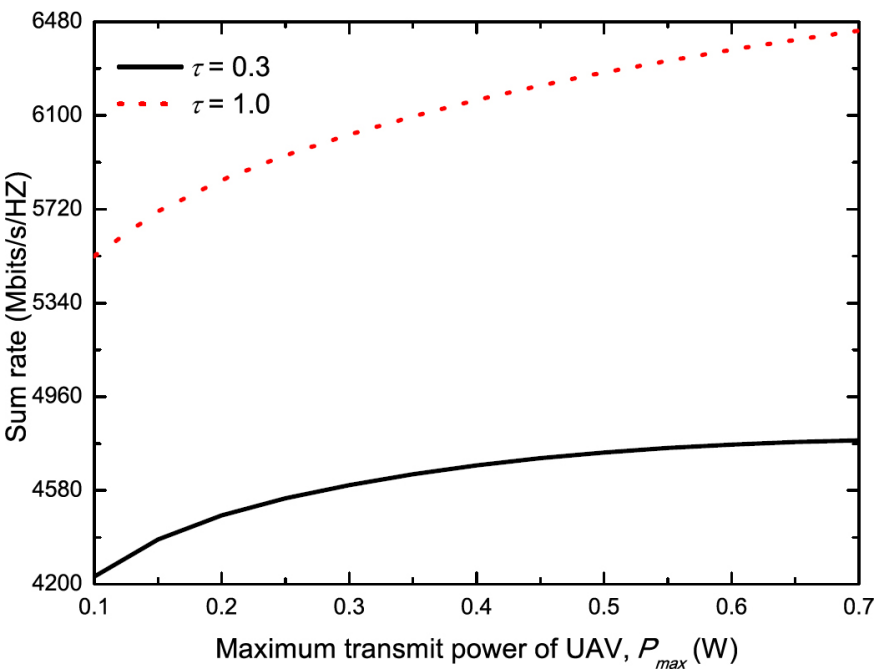

Fig. 4. The effect of maximum UAV transmit power $P_{\max }$ of on sum rate

Meanwhile, it also results in a high path loss. As $H_{U}$ is relative small, the positive effect of antenna gain on link rates is more than the negative effect of path loss, and thus the sum rate increases as $H_{U}$ increases. As $H_{U}$ further increase, they have the same effect on the link rates, and thus the sum rate remains unchanged. As $H_{U}$ proceeds to increase, the negative effect is more than the positive effect, and thus the sum rate reduces.

\section{Effect of $P_{\max }$ On Sum Rate}

Finally, we explore the effect of maximum UAV transmit power $P_{\max }$ on the sum rate for the scenario of $\tau=\{0.3,1.0\}$. We summarize in Fig. 4 how the sum rate varies with $P_{\max }$. We can observe from Fig. 4 that for each fixed setting of $\tau$, the growing speed of sum rate is from fast to slow. This is because an increase of $P_{\max }$ could lead to the increase of optimal UAV transmit power, which has two-fold effect on the sum rate performance. As the optimal UAV transmit pow is relative small, an increase of the optimal UAV transmit power results in the increase of link rates. As the optimal UAV transmit pow continues to increase, the severe interference results in the decrease of the growing speed for link rates.

\section{CONClusion}

This paper explored the sum rate maximization for the cellular-connected UAV swarm communications. The sum rate maximization problem was formulated as a nonlinear and nonconvex optimization problem, which is generally challenging to solve. Based on the KKT conditions and the standard interference function results, we propose an iterative algorithm to solve it. This algorithm was proved to converge to a globally optimal solution. Simulation results indicate that we can find an optimal antenna beamwidth for maximizing the sum rate, and an increase of maximum UAV transmit power leads to the increase of sum rate.

Acknowledgment: This work was supported by the European Union's Horizon 2020 Research and Innovation Program through the 5G!Drones Project under Grant No. 857031, by 
the Academy of Finland 6Genesis project under Grant No. 318927 , by the Academy of Finland CSN project under Grant No. 311654, by the NSFC under Grant No. 61962033, by the Anhui Province project under Grant No. 1808085MF165, gxgwfx2019060, and KJ2019A0643, by the Yunnan Province project under Grant No.2018FH001-010, and by the CHZU project under Grant No. 2020qd16 and zrjz2019011.

\section{REFERENCES}

[1] N. H. Motlagh, T. Taleb, and O. Arouk, "Low-altitude unmanned aerial vehicles-based internet of things services: Comprehensive survey and future perspectives," IEEE Internet of Things J., vol. 3, no. 6, pp. 899 922, Dec. 2016

[2] N. H. Motlagh, M. Bagaa, and T. Taleb, "Energy and delay aware task assignment mechanism for UAV-based IoT platform," IEEE Internet Things J., vol. 6, no. 4, p. 6523-6536, Aug. 2019.

[3] B. Yang, T. Taleb, Z. Wu, and L. Ma, "Spectrum sharing for secrecy performance enhancement in D2D-enabled UAV networks," IEEE Network, vol. 34 , no. 6 , pp. 156-163, Nov./Dec. 2020

[4] B. Yang, T. Taleb et al., "Mode selection and cooperative jamming for covert communication in D2D underlaid UAV networks," IEEE Network (Accepted), 2020

[5] O. Bekkouche, K. Samdanis, M. Bagaa, and T. Taleb, "A servicebased architecture for enabling UAV enhanced network services," IEEE Network, vol. 34, no. 4, pp. 328-335, 2020.

[6] M. Maiouak and T. Taleb, "Dynamic maps for automated driving and UAV geofencing," IEEE Wireless Commun., vol. 26, no. 4, pp. 54-59, 2019.

[7] L. Hu, Y. Tian, J. Yang, T. Taleb et al., "Ready player one: UAVclustering-based multi-task offloading for vehicular VR/AR gaming," IEEE Network, vol. 33, no. 3, pp. 42-48, 2019.

[8] H. Hellaoui, O. Bekkouche, M. Bagaa, and T. Taleb, "Aerial control system for spectrum efficiency in UAV-to-cellular communications," IEEE Communications Magazine, vol. 56, no. 10, pp. 108-113, 2018.

[9] V. V. Chetlur and H. S. Dhillon, "Downlink coverage analysis for a finite $3 \mathrm{~d}$ wireless network of unmanned aerial vehicles," IEEE Trans. Commun., vol. 65, no. 10, p. 4543-4558, Jun. 2017.

[10] X. Zhang and L. Duan, "Fast deployment of UAV networks for optimal wireless coverage," IEEE Trans. Mobile Comput., vol. 18, no. 3, p. 588-601, Mar. 2019.

[11] Y. Zeng, X. Xu, and R. Zhang, "Trajectory design for completion time minimization in UAV-enabled multicasting," IEEE Trans. Wireless Commun., vol. 17, no. 4, p. 2233-2246, Apr. 2018.

[12] C. Shen, T.-H. Chang, J. Gong, Y. Zeng, and R. Zhang, "Multi-UAV interference coordination via joint trajectory and power control," IEEE Trans. Sig. Proc., vol. 68, pp. 843-858, Jan. 2020.

[13] C. You and R. Zhang, "3D trajectory optimization in Rician fading for UAV-enabled data harvesting," IEEE Trans. Wireless Commun., vol. 18, no. 6, p. 3192-3207, Jun. 2019.

[14] H. He, S. Zhang, Y. Zeng, and R. Zhang, "Joint altitude and beamwidth optimization for uav-enabled multiuser communications," IEEE Commun. Lett., vol. 22, no. 2, p. 344-347, Feb. 2018.

[15] M. Hua, L. Yang, Q. Wu, and A. L. Swindlehurst, "3D UAV trajectory and communication design for simultaneous uplink and downlink transmission," 2020. [Online]. Available: https://arxiv.org/abs/2001.00351

[16] Q. Wu and R. Zhang, "Common throughput maximization in UAVenabled OFDMA systems with delay consideration," IEEE Trans. Commun., vol. 66, no. 12, pp. 6614-6627, Dec. 2018.

[17] H. Wu, X. Tao, N. Zhang, and X. Shen, "Cooperative UAV cluster assisted terrestrial cellular networks for ubiquitous coverage," IEEE J. Sel. Areas Commun., vol. 36, no. 9, p. 2045-2058, Aug. 2018.

[18] S. Zhang, Y. Zeng, and R. Zhang, "Cellular-enabled UAV communication: A connectivity-constrained trajectory optimization perspective," IEEE Trans. Commun., vol. 67, no. 3, p. 2580-2604, Mar. 2019.

[19] W. Mei and R. Zhang, "Cooperative downlink interference transmission and cancellation for cellular-connected UAV: A divide-and-conquer approach," IEEE Trans. Commun., vol. 68, no. 2, pp. 1297-1311, Feb. 2020.

[20] S. Zhang, H. Zhang, B. Di, and L. Song, "Cellular UAV-to-X communications: Design and optimization for multi-UAV networks," IEEE Trans. Wireless Commun., vol. 18, no. 2, p. 1346-1359, Feb. 2019.
[21] Y. Li, G. Feng, M. Ghasemiahmadi, and L. Cai, "Power allocation and 3-D placement for floating relay supporting indoor communications," IEEE Trans. Mobile Comput., vol. 18, no. 3, p. 618-631, Mar. 2019.

[22] B. Yang, T. Taleb, Y. Shen, X. Jiang, and W. Yang, "Performance, fairness and tradeoff in uav swarm underlaid mmwave cellular networks with directional antennas," IEEE Trans. Wireless Commun., 2020. [Online]. Available: https://doi.org/10.1109/TWC.2020.3041800

[23] A. Goldsmith, "Wireless communications," in Cambridge University Press, 2005

[24] C. A. Balanis, "Antenna theory: Analysis and design, 3rd ed," in WileyInterscience, 2005.

[25] R. D. Yates, "A framework for uplink power control in cellular radio systems," IEEE J. Sel. Areas Commun., vol. 13, no. 7, p. 1341-1348, Sep. 1995 